\title{
A stochastic model for an urea decomposition system
}

\author{
VSS Yadavalli|k MAE Muller|" \\ Received: 19 April 2005; Revised: 24 June 2005; Accepted: 28 July 2005
}

\begin{abstract}
Availability is an important measure in describing the performance of a system. The availability of a decomposition process in an urea production system in the fertilizer industry is considered in this paper. The system contains four subsystems and is supported by a standby unit. An estimation study of the steady state availability of the system is performed and illustrated by means of a numerical example.
\end{abstract}

Key words: Urea decomposition system, availability, steady state availability, interval estimation.

\section{Introduction}

The role and importance of (being able to measure) reliability has been a core issue in the Engineering industry over the last three decades. Reliability is of importance to both manufacturers and consumers. From the point of view of consumers and manufacturers, reliability provides quality and vice versa. The reliability measure is also very important, as improvement in reliability of a system is often achieved through quality analyses. While this measure is of great importance in industry, there are many situations where continuous failure free performance of a system, though desirable, may not be absolutely necessary. For instance, in a production industry possible interruptions may occur and can be tolerated. In such situations it may be eminently reasonable to introduce another measure called 'availability,' which denotes the probability that the system is functioning at any time instant.

In the process industry, like the fertilizer industry, one encounters many processes like synthesis decomposition, crystallization, prilling and recovery. See, for example, Kumar et al. (1991) and the UN Fertelizer Manual (1967).

\footnotetext{
${ }^{*}$ Corresponding author: Department of Industrial and Systems Engineering, University of Pretoria, 0002, South Africa, email: sarma.yadavalli@up.ac.za

${ }^{\dagger}$ Department of Industrial and Systems Engineering, University of Pretoria, Pretoria, 0002, South Africa, and Department of Statistics, University of Namibia, Namibia
} 
In an urea decomposition system a gas liquid mixture (urea, $\mathrm{NH}_{3}, \mathrm{CO}_{2}$, Biuret) flows from a reactor at $126^{\circ} \mathrm{C}$ into the upper part of a high-pressure decomposer where the flushed gasses are separated. The liquid falls through a sieve plate, which comes in contact with high temperature gas available from a boiler and a falling film heater. The process is repeated in a low-pressure absorber. The solution is further heated to $165^{\circ} \mathrm{C}$ in a falling film heater, which reduces the Biuret formation and hydrolysis of urea (see Figure 1 for the layout of a typical urea decomposition plant).

The overhead gasses from the high-pressure decomposition then go to the high-pressure absorber cooler. The liquid flows to the top of a low-pressure absorber and is cooled in a heat exchanger. Additional flushing of the solution takes place in the upper part of the low-pressure absorber so as to reduce the solution pressure from 17.5 to $2.5 \mathrm{~kg} / \mathrm{cm}^{2}$. The low-pressure absorber has four sieve trays and a packed bed. In the packed bed, the remaining ammonia is stripped off by means of $\mathrm{CO}_{2}$ gas.

The overhead gases then go to the low-pressure absorber cooler, in which the pressure is controlled at $2.2 \mathrm{~kg} / \mathrm{cm}^{2}$. Most of the excess ammonia and carbonate is separated from the solution flowing to the gas separator. The gas separator has two parts:

1. the upper part functions at $105^{\circ} \mathrm{C}$ and $0.3 \mathrm{~kg} / \mathrm{cm}^{2}$ and here the remaining small amounts of ammonia and $\mathrm{CO}_{2}$ are recovered by reducing the pressure; the heat of the solution is enough to vaporize these gasses.

2. The lower part comprises a packed section at $110^{\circ} \mathrm{C}$ and atmospheric pressure.

Air containing a small amount of ammonia and $\mathrm{CO}_{2}$ is fed off from the gas absorber by an on/off gas blower so as to remove the remaining small amounts of ammonia and $\mathrm{CO}_{2}$ present in the solution. Off gasses from the lower and upper parts are mixed and led to the off-gas condenser. The urea solution concentrated to $70-75 \%$ is finally fed to a crystallizer.

\section{System description and notation}

The complex system (urea plant in Figure 1) described in the previous section consists of four subsystems connected in series. The following assumptions are required to describe the system:

1. Subsystem $\left(A_{i}\right)$ comprises two units. Unit $A_{1}$ is the boiler for the high-pressure absorber and $A_{2}$ is the falling filter heater for the low-pressure absorber. This subsystem $\left(A_{i}\right)$ fails by failure of $A_{1}$ or $A_{2}$.

2. Subsystem $B_{i}$ comprises two units in series. Unit $B_{1}$ is called the high-pressure absorber and unit $B_{2}$ is called the low-pressure absorber. Failure of either causes complete failure of the system.

3. Subsystem D, the gas separator, comprises only one unit, arranged in series with $B_{1}$ and $B_{2}$. Failure of unit D causes complete failure of the system. 


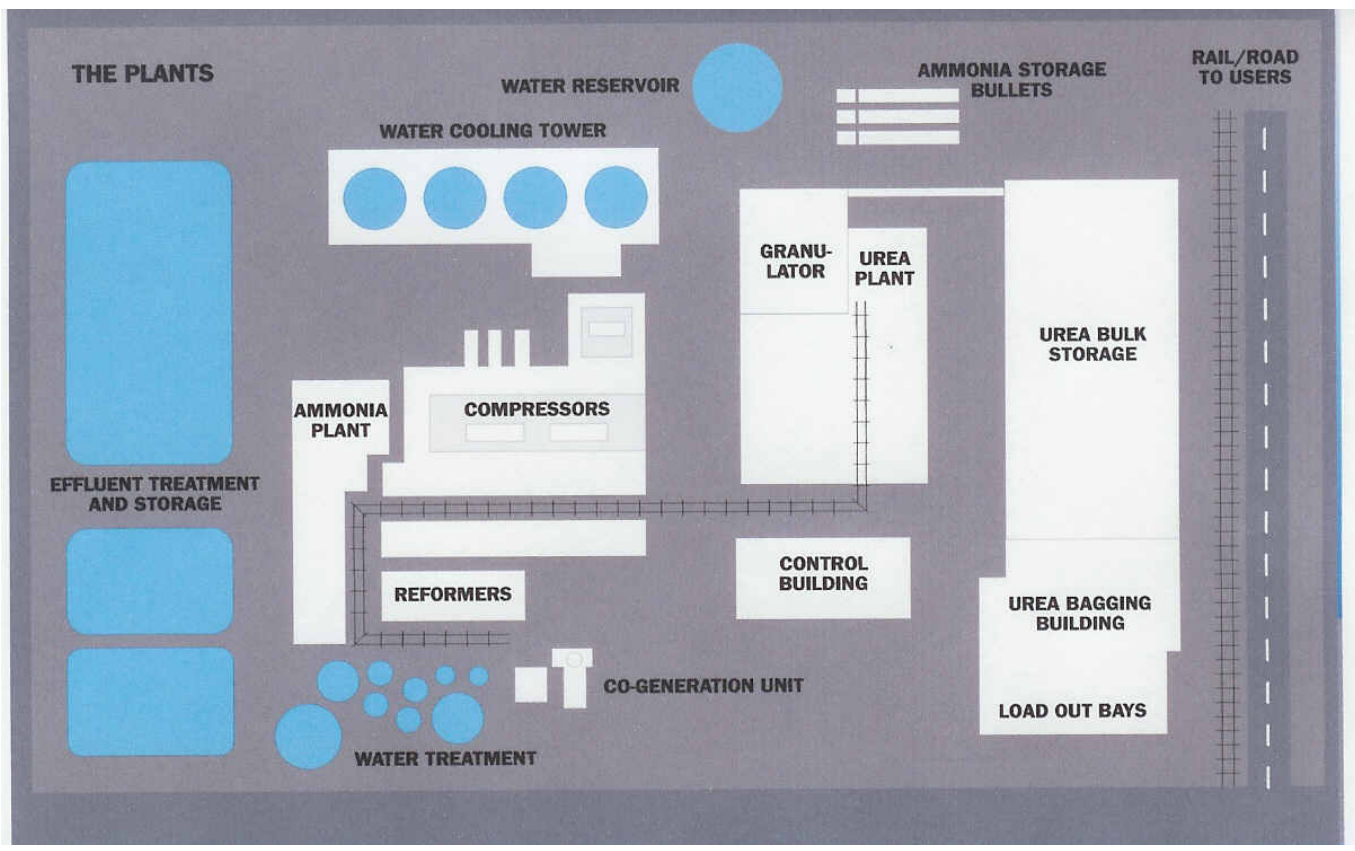

Figure 1: Plan of an urea plant (by courtesy of Balance Kapuni, South Taranaki, New Zealand),

4. Subsystem $E_{i}$ the heat exchanger comprises one unit and another in standby mode. Failure occurs only when both units fail.

5. The life time of the units $\left(A_{i}, B_{i}, D, E_{i} ; \quad i=1,2\right)$ are exponentially distributed random variables with parameters $\lambda_{i} ; \quad i=1,2, \ldots, 6$.

6. The repair time of the units are exponentially distributed random variables with parameters $\mu_{j} ; j=1,2, \ldots, 6$.

7. Each unit is as good as new after repair.

8. Spare parts and the repair facility are always available.

9. The standby unit in $\mathrm{E}$ is of the same nature and capacity as the operating active unit.

10. The repair is performed at regular time intervals or at the time of failure. The repair includes replacement as well.

11. There is no common failure among subsystems.

12. State 0 indicates the operating state without using the standby unit and state 6 indicates the operating state using the standby state in subsystem E.

13. $E_{1}$ is the state of the system running at full capacity with a standby unit in subsystem E. 


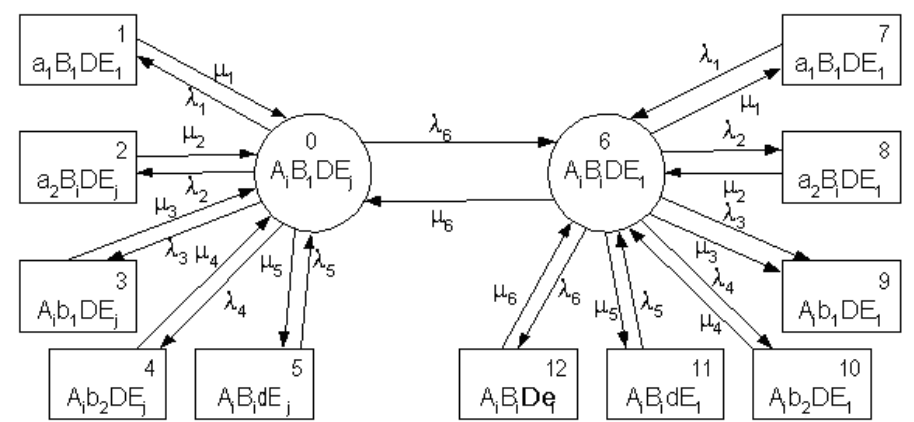

Figure 2: The state transition diagram.

\section{Availability analysis of the system}

Let $P_{i}(t)$ denote the probability that the system is in state $i$ at time $t$ and let $P_{i}=$ $\lim _{t \rightarrow \infty} P_{i}(t)$.

Using Figure 2, the flow balance technique [2] is applied to find the steady state probabilities $\left(P_{i}\right)$ :

$$
\begin{aligned}
\left(\sum_{i=1}^{6} \lambda_{i}\right) P_{0} & =\sum_{i=1}^{6} \mu_{i} P_{i}, \\
\left(\sum_{i=1}^{6} \lambda_{i}+\mu_{6}\right) P_{6} & =\sum_{i=1}^{6} \mu_{i} P_{i+6}+\lambda_{6} P_{0}, \\
\sum_{i=1}^{5} \mu_{i} P_{i} & =\sum_{i=1}^{5} \lambda_{i} P_{0} ; \quad i=1,2, \ldots, 6 \\
\sum_{i=1}^{6} \mu_{i} P_{i+6} & =\sum_{i=1}^{6} P_{6} ; \quad i=1,2, \ldots, 6 \\
\text { and } \sum_{i=1}^{6} P_{i} & =1 .
\end{aligned}
$$

Solving the system of equations (1)-(5), we obtain $P_{i}$. The system measure $A_{\infty}$, namely the probability that the system is up over the long run, may be obtained as

$$
A_{\infty}=P_{0}+P_{6}=\frac{1+\frac{\lambda_{6}}{\mu_{6}}}{1+\left(1+\frac{\lambda_{6}}{\mu_{6}}\right) \sum_{i=1}^{6} \frac{\lambda_{i}}{\mu_{i}}} .
$$

\section{Interval estimation for $A_{\infty}$}

Let $X_{i 1}, X_{i 2}, \ldots, X_{i n}(i=1,2, \ldots, 6)$ be random samples of size $n$, each drawn from different exponential populations with failure rates $\lambda_{i}$. Similarly, let $y_{i 1}, y_{i 2}, \ldots, y_{i n}(i=$ $1,2, \ldots, 6)$ be random samples, each drawn from exponential populations with parameters 


\begin{tabular}{llllllllll}
\hline$\alpha_{1}=\alpha_{2}$ & 0 & 0 & 0 & 0.001 & 0.001 & 0.001 & 0.005 & 0.005 & 0.005 \\
$\alpha_{3}=\alpha_{4}=\alpha_{5}$ & 0 & 0.005 & 0.01 & 0 & 0.005 & 0.01 & 0 & 0.005 & 0.01 \\
\hline$\alpha_{6}=0$ & 1 & 0.90909 & 0.83333 & 0.99602 & 0.90578 & 0.83056 & 0.98037 & 0.89284 & 0.81967 \\
$\alpha_{6}=0.001$ & 0.99998 & 0.90907 & 0.83332 & 0.99600 & 0.90578 & 0.83055 & 0.98037 & 0.89284 & 0.81966 \\
$\alpha_{6}=0.002$ & 0.99994 & 0.90903 & 0.83328 & 0.99595 & 0.90574 & 0.83052 & 0.98033 & 0.89280 & 0.81962 \\
$\alpha_{6}=0.003$ & 0.99985 & 0.90897 & 0.83323 & 0.99587 & 0.90568 & 0.83046 & 0.98025 & 0.89274 & 0.81957 \\
$\alpha_{6}=0.004$ & 0.99974 & 0.90888 & 0.83316 & 0.99577 & 0.90559 & 0.83039 & 0.98015 & 0.89266 & 0.81950 \\
$\alpha_{6}=0.005$ & 0.99961 & 0.90877 & 0.83306 & 0.99563 & 0.90548 & 0.83029 & 0.98002 & 0.89254 & 0.81941 \\
$\alpha_{6}=0.006$ & 0.99944 & 0.90863 & 0.83294 & 0.99546 & 0.90534 & 0.83018 & 0.97985 & 0.89241 & 0.81929 \\
$\alpha_{6}=0.007$ & 0.99924 & 0.90846 & 0.83280 & 0.99526 & 0.90517 & 0.83004 & 0.97966 & 0.89255 & 0.81916 \\
$\alpha_{6}=0.008$ & 0.99901 & 0.90827 & 0.83264 & 0.99503 & 0.90498 & 0.82988 & 0.97944 & 0.89207 & 0.81901 \\
$\alpha_{6}=0.009$ & 0.99875 & 0.90806 & 0.83247 & 0.99478 & 0.90477 & 0.82970 & 0.97919 & 0.89186 & 0.81883 \\
\hline
\end{tabular}

Table 1: Availability measure, $A_{\infty}\left(\beta_{1}=\beta_{2}=0.5 ; \beta_{3}=\beta_{4}=0.2 ; \beta_{5}=0.1 ; \beta_{6}=0.25\right)$.

\begin{tabular}{llllllllll}
\hline$\alpha_{1}=\alpha_{2}$ & 0 & 0 & 0 & 0.001 & 0.001 & 0.001 & 0.005 & 0.005 & 0.005 \\
$\alpha_{3}=\alpha_{4}=\alpha_{5}$ & 0 & 0.005 & 0.01 & 0 & 0.005 & 0.01 & 0 & 0.005 & 0.01 \\
\hline$\alpha_{6}=0$ & 1 & 0.90900 & 0.83333 & 0.99668 & 0.90634 & 0.83102 & 0.98361 & 0.89552 & 0.82192 \\
$\alpha_{6}=0.001$ & 0.99998 & 0.90908 & 0.83332 & 0.99666 & 0.90633 & 0.83101 & 0.98359 & 0.89551 & 0.82191 \\
$\alpha_{6}=0.002$ & 0.99994 & 0.90904 & 0.83329 & 0.99661 & 0.90629 & 0.83098 & 0.98355 & 0.89547 & 0.82187 \\
$\alpha_{6}=0.003$ & 0.99986 & 0.90894 & 0.83323 & 0.99654 & 0.90623 & 0.83093 & 0.98347 & 0.89541 & 0.82182 \\
$\alpha_{6}=0.004$ & 0.99975 & 0.90888 & 0.83316 & 0.99643 & 0.90614 & 0.83085 & 0.98336 & 0.89532 & 0.82175 \\
$\alpha_{6}=0.005$ & 0.99961 & 0.90877 & 0.83316 & 0.99629 & 0.90602 & 0.83075 & 0.98323 & 0.89521 & 0.82165 \\
$\alpha_{6}=0.006$ & 0.99944 & 0.90863 & 0.83306 & 0.99612 & 0.90588 & 0.83064 & 0.98306 & 0.89507 & 0.82154 \\
$\alpha_{6}=0.007$ & 0.99924 & 0.90846 & 0.83294 & 0.99592 & 0.90572 & 0.83050 & 0.98287 & 0.89491 & 0.82140 \\
$\alpha_{6}=0.008$ & 0.99901 & 0.90827 & 0.83280 & 0.99569 & 0.90553 & 0.83034 & 0.98265 & 0.89473 & 0.82125 \\
$\alpha_{6}=0.009$ & 0.99875 & 0.90806 & 0.83247 & 0.99544 & 0.90532 & 0.83016 & 0.98240 & 0.89452 & 0.82107 \\
\hline
\end{tabular}

Table 2: Availability measure, $A_{\infty}\left(\beta_{1}=\beta_{2}=0.6 ; \beta_{3}=\beta_{4}=0.2 ; \beta_{5}=0.1 ; \beta_{6}=0.25\right)$.

\begin{tabular}{llllllllll}
\hline$\alpha_{1}=\alpha_{2}$ & 0 & 0 & 0 & 0.001 & 0.001 & 0.001 & 0.005 & 0.005 & 0.005 \\
$\alpha_{3}=\alpha_{4}=\alpha_{5}$ & 0 & 0.005 & 0.01 & 0 & 0.005 & 0.01 & 0 & 0.005 & 0.01 \\
\hline$\alpha_{6}=0$ & 1 & 0.92308 & 0.85714 & 0.99715 & 0.92065 & 0.85505 & 0.98592 & 0.91106 & 0.84677 \\
$\alpha_{6}=0.001$ & 0.99998 & 0.92306 & 0.85713 & 0.99714 & 0.92064 & 0.85504 & 0.9859 & 0.91105 & 0.84676 \\
$\alpha_{6}=0.002$ & 0.99994 & 0.92302 & 0.85710 & 0.99709 & 0.9206 & 0.85500 & 0.98585 & 0.91101 & 0.84673 \\
$\alpha_{6}=0.003$ & 0.99986 & 0.92296 & 0.85704 & 0.99701 & 0.92053 & 0.85494 & 0.98578 & 0.91094 & 0.84667 \\
$\alpha_{6}=0.004$ & 0.99975 & 0.92286 & 0.85696 & 0.9969 & 0.92044 & 0.85486 & 0.98567 & 0.91085 & 0.84659 \\
$\alpha_{6}=0.005$ & 0.99961 & 0.92274 & 0.85685 & 0.99676 & 0.92032 & 0.85476 & 0.98553 & 0.91074 & 0.84649 \\
$\alpha_{6}=0.006$ & 0.99944 & 0.9226 & 0.92260 & 0.99659 & 0.92017 & 0.84637 & 0.98537 & 0.91060 & 0.84637 \\
$\alpha_{6}=0.007$ & 0.99924 & 0.92243 & 0.92243 & 0.99639 & 0.92000 & 0.84623 & 0.98517 & 0.91043 & 0.84623 \\
$\alpha_{6}=0.008$ & 0.99901 & 0.92223 & 0.92223 & 0.99617 & 0.91981 & 0.84606 & 0.98495 & 0.91024 & 0.84606 \\
$\alpha_{6}=0.009$ & 0.99875 & 0.92201 & 0.92201 & 0.99591 & 0.91969 & 0.84588 & 0.98470 & 0.91003 & 0.84588 \\
\hline
\end{tabular}

Table 3: Availability measure, $A_{\infty}\left(\beta_{1}=\beta_{2}=0.7 ; \beta_{3}=\beta_{4}=0.3 ; \beta_{5}=0.1 ; \beta_{6}=0.25\right)$. 


\begin{tabular}{llllllllll}
\hline$\alpha_{1}=\alpha_{2}$ & 0 & 0 & 0 & 0.001 & 0.001 & 0.001 & 0.005 & 0.005 & 0.005 \\
$\alpha_{3}=\alpha_{4}=\alpha_{5}$ & 0 & 0.005 & 0.01 & 0 & 0.005 & 0.01 & 0 & 0.005 & 0.01 \\
\hline$\alpha_{6}=0$ & 0.99998 & 0.93023 & 0.86957 & 0.99751 & 0.92807 & 0.86768 & 0.98765 & 0.91954 & 0.86022 \\
$\alpha_{6}=0.001$ & 0.99994 & 0.93022 & 0.86955 & 0.99749 & 0.92806 & 0.86767 & 0.98764 & 0.91953 & 0.8602 \\
$\alpha_{6}=0.002$ & 0.99986 & 0.93018 & 0.86952 & 0.99744 & 0.92802 & 0.86763 & 0.98759 & 0.91949 & 0.86017 \\
$\alpha_{6}=0.003$ & 0.99975 & 0.93011 & 0.86946 & 0.99736 & 0.92795 & 0.86757 & 0.98752 & 0.91942 & 0.86011 \\
$\alpha_{6}=0.004$ & 0.99975 & 0.93001 & 86937 & 0.99726 & 0.92786 & 0.86749 & 0.98741 & 0.91933 & 0.86003 \\
$\alpha_{6}=0.005$ & 0.99961 & 0.92989 & 0.8627 & 0.99712 & 0.92774 & 0.86738 & 0.98727 & 0.91921 & 0.85992 \\
$\alpha_{6}=0.006$ & 0.99944 & 0.92975 & 0.86914 & 0.99695 & 0.92759 & 0.86726 & 0.98711 & 0.91906 & 0.858 \\
$\alpha_{6}=0.007$ & 0.99924 & 0.92957 & 0.86899 & 0.99675 & 0.92742 & 0.86711 & 0.98691 & 0.9189 & 0.8597 \\
$\alpha_{6}=0.008$ & 0.99901 & 0.92937 & 0.86882 & 0.99652 & 0.92722 & 0.86893 & 0.98669 & 0.9187 & 0.85948 \\
$\alpha_{6}=0.009$ & 0.99875 & 0.92915 & 0.86862 & 0.99626 & 0.927 & 0.86674 & 0.98644 & 0.91848 & 0.85929 \\
\hline
\end{tabular}

Table 4: Availability measure, $A_{\infty}\left(\beta_{1}=\beta_{2}=0.8 ; \beta_{3}=\beta_{4}=0.4 ; \beta_{5}=0.1 ; \beta_{6}=0.25\right)$.

\begin{tabular}{llllllllll}
\hline$\alpha_{1}=\alpha_{2}$ & 0 & 0 & 0 & 0.001 & 0.001 & 0.001 & 0.005 & 0.005 & 0.005 \\
$\alpha_{3}=\alpha_{4}=\alpha_{5}$ & 0 & 0.005 & 0.01 & 0 & 0.005 & 0.01 & 0 & 0.005 & 0.01 \\
\hline$\alpha_{6}=0$ & 1 & 0.93458 & 0.87719 & 0.99778 & 0.93264 & 0.87549 & 0.98901 & 0.92497 & 0.86873 \\
$\alpha_{6}=0.001$ & 0.99998 & 0.93457 & 0.87718 & 0.99777 & 0.93263 & 0.87547 & 0.989 & 0.92496 & 0.86871 \\
$\alpha_{6}=0.002$ & 0.99994 & 0.93452 & 0.87714 & 0.99772 & 0.93259 & 0.87544 & 0.98895 & 0.92492 & 0.86868 \\
$\alpha_{6}=0.003$ & 0.99986 & 0.93446 & 0.87708 & 0.99764 & 0.93252 & 0.87538 & 0.98887 & 0.92485 & 0.86862 \\
$\alpha_{6}=0.004$ & 0.99975 & 0.93436 & 0.871 & 0.99753 & 0.93242 & 0.87529 & 0.98876 & 0.92476 & 0.86854 \\
$\alpha_{6}=0.005$ & 0.99961 & 0.93424 & 0.87689 & 0.99739 & 0.9323 & 0.87519 & 0.98863 & 0.92464 & 0.86843 \\
$\alpha_{6}=0.006$ & 0.99944 & 0.93409 & 0.87676 & 0.99722 & 0.93215 & 0.87506 & 0.98846 & 0.92449 & 0.8683 \\
$\alpha_{6}=0.007$ & 0.99924 & 0.93391 & 0.87661 & 0.99702 & 0.93198 & 0.8749 & 0.98827 & 0.92432 & 0.86815 \\
$\alpha_{6}=0.008$ & 0.99901 & 0.93371 & 0.87643 & 0.9968 & 0.93178 & 0.87473 & 0.98804 & 0.92413 & 0.86798 \\
$\alpha_{6}=0.009$ & 0.99875 & 0.93349 & 0.87623 & 0.99654 & 0.93156 & 0.87453 & 0.98779 & 0.92391 & 0.86778 \\
\hline
\end{tabular}

Table 5: Availability measure, $A_{\infty}\left(\beta_{1}=\beta_{2}=0.9 ; \beta_{3}=\beta_{4}=0.5 ; \beta_{5}=0.1 ; \beta_{6}=0.25\right)$.

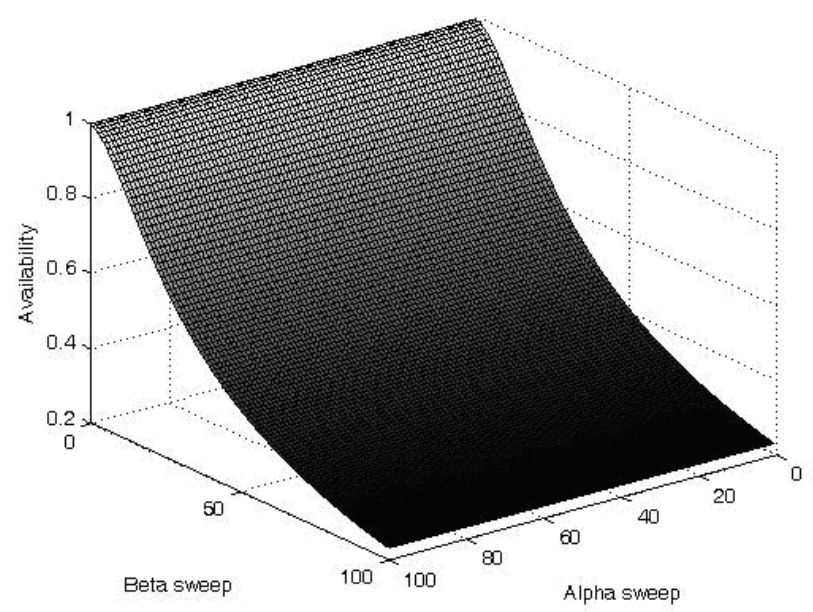

Figure 3: Availability measure, $A_{\infty}$, for different values of $\alpha$ (failure-free operation time) and $\beta$ (repair time) values. 
$\mu_{i}$. Since $\lambda_{i}$ is a parameter of the exponential distribution, an estimate may be found for $\lambda_{i}$ or for $1 / \lambda_{i}=\alpha_{i}$ (say), which is equal to the mean value of failure-free time. Let

$$
\alpha_{i}=\frac{1}{\lambda_{i}} \quad \text { and } \quad \beta_{i}=\frac{1}{\mu_{i}} .
$$

Tables 1 to 5 and Figure 3 show values of the above availability function, $A_{\infty}$ for different values of the parameters $\alpha_{i}$ and $\beta_{i}$. Then the maximum likelihood estimates (MLE) of $\alpha_{i}$ and $\beta_{i}$ are given by

$$
\frac{1}{n} \sum_{j=1}^{n} X_{i j}=\bar{x}_{i} \quad \text { and } \quad \frac{1}{n} \sum_{j=1}^{n} Y_{i j}=\bar{y}_{i}
$$

respectively. Hence

$$
\hat{A}_{\infty}=\frac{1+\bar{y}_{6} / x_{6}}{1+\left(1+\bar{y}_{6} / \bar{x}_{6}\right) \sum_{i=1}^{6} \bar{y}_{i} / \bar{x}_{i}} .
$$

Applying the multivariate central limit theorem (See, for example, Rao (1973)), yields $\sqrt{n}(\bar{x}-\theta) \stackrel{D}{\longrightarrow} N_{6}(0, \Sigma)$ as $n \rightarrow \infty$, where $\bar{x}=\left(\bar{x}_{1}, \bar{x}_{2}, \bar{x}_{3}, \bar{x}_{4}, \bar{x}_{5}, \bar{x}_{6}, \bar{y}_{1}, \bar{y}_{2}, \bar{y}_{3}, \bar{y}_{4}, \bar{y}_{5}, \bar{y}_{6}\right)$ and $\theta=\left(\alpha_{1}, \alpha_{2}, \alpha_{3}, \alpha_{4}, \alpha_{5}, \alpha_{6}, \beta_{1}, \beta_{2}, \beta_{3}, \beta_{4}, \beta_{5}, \beta_{6}\right)$. The dispersion matrix $\Sigma=\left(\sigma_{i j}\right)_{12 \times 12}$ is given by $\Sigma=\operatorname{diag}\left(\alpha_{1}^{2}, \ldots, \alpha_{6}^{2}, \beta_{1}^{2}, \ldots, \beta_{6}^{2}\right)$. Hence, $\sqrt{n}\left(\hat{A}_{\infty}-A_{\infty}\right) \stackrel{D}{\longrightarrow} N_{6}\left(0, \sigma^{2}(\theta)\right)$, where

$$
\sigma^{2}(\theta)=\sum_{i=1}^{6}\left(\frac{\delta A_{\infty}}{\delta \alpha_{i}}\right)^{2} \delta_{i i}+\sum_{i=1}^{6}\left(\frac{\delta A_{\infty}}{\delta \beta_{i}}\right)^{2} \delta_{i i}
$$

and where $\hat{\theta}=\left(\bar{\alpha}_{1}, \bar{\alpha}_{2}, \bar{\alpha}_{3}, \bar{\alpha}_{4}, \bar{\alpha}_{5}, \bar{\alpha}_{6}, \bar{\beta}_{1}, \bar{\beta}_{2}, \bar{\beta}_{3}, \bar{\beta}_{4}, \bar{\beta}_{5}, \bar{\beta}_{6}\right)$, which entails that $\hat{\sigma}^{2}=\sigma^{2}(\theta)$ is a consistent estimator of $\sigma^{2}(\theta)$. Therefore by Slutzky's theorem (see, for example, Slutzky (1928)),

$$
\frac{\sqrt{n}\left(\hat{A}_{\infty}-A_{\infty}\right)}{\hat{\sigma}} \stackrel{D}{\longrightarrow} N(0,1) \text { as } n \rightarrow \infty
$$

or,

$$
P\left[-\kappa \frac{\alpha}{2} \leq \frac{\sqrt{n}\left(\hat{A}_{\infty}-A_{\infty}\right)}{\hat{\sigma}} \leq \kappa \frac{\alpha}{2}\right]=1-\alpha
$$

\section{$5 \quad$ Numerical illustration}

The $\alpha=95 \%$ and $\alpha=99 \%$ confidence intervals for different simulated samples are presented in Table 6. Observe that the steady state availability decreases if $n$ increases.

\section{Conclusion}

The availability of equipment used for the decomposition process in a urea production system was considered in this paper. The system consists of four subsystems, with a standby unit in one of the sub-systems. The long run availability of the system is calculated, and asymptotic confidence limits are obtained for the steady-state availability. The results clearly show that, as the repair time increases, the steady state availability decreases. 


\begin{tabular}{rrcc}
\hline & & $\alpha=95 \%$ & $\alpha=99 \%$ \\
\hline$n=100$ & 20 & $(0.79414 ; 0.96586)$ & $(0.76702 ; 0.99298)$ \\
& 40 & $(0.62674 ; 0.78366)$ & $(0.60196 ; 0.80824)$ \\
& 60 & $(0.54593 ; 0.68317)$ & $(0.52533 ; 0.69537)$ \\
$n=200$ & 80 & $(0.50775 ; 0.61515)$ & $(0.48552 ; 0.62088)$ \\
& 100 & $(0.47816 ; 0.56924)$ & $(0.45556 ; 0.57544)$ \\
& 20 & $(0.81928 ; 0.94072)$ & $(0.80008 ; 0.95992)$ \\
& 40 & $(0.64986 ; 0.76074)$ & $(0.63234 ; 0.77826)$ \\
$n=2000$ & 60 & $(0.57289 ; 0.66421)$ & $(0.55843 ; 0.67867)$ \\
& 80 & $(0.52347 ; 0.59943)$ & $(0.51147 ; 0.61143)$ \\
& 100 & $(0.49148 ; 0.55592)$ & $(0.48128 ; 0.56612)$ \\
& 20 & $(0.86080 ; 0.89920)$ & $(0.85468 ; 0.90532)$ \\
& 40 & $(0.68790 ; 0.72270)$ & $(0.67998 ; 0.73062)$ \\
& 60 & $(0.60409 ; 0.63301)$ & $(0.59953 ; 0.63757)$ \\
& 80 & $(0.54945 ; 0.57345)$ & $(0.54567 ; 0.57723)$ \\
& 100 & $(0.51356 ; 0.53384)$ & $(0.51032 ; 0.53708)$ \\
\hline
\end{tabular}

Table 6: Confidence limits for $A_{\infty}$ (For $\alpha_{1}=\alpha_{2}=0 ; \alpha_{3}=\alpha_{4}=\alpha_{5}=0 ; \alpha_{6}=0.001 \beta_{1}=\beta_{2}=$ $\left.0.5 ; \beta_{3}=\beta_{4}=0.2 ; \beta_{5}=0.1 ; \beta_{6}=0.25\right)$.

\section{Acknowledgements}

The authors wish to thank the two anonymous referees for their valuable comments.

\section{References}

[1] Kumar D, Pandey PC \& Singh L, 1991, Process design for a crystallization system in the urea fertilizer industry, Microelectronics and Reliability, 31(1), pp. 855-859.

[2] Ravindrasn A, Phillips DJ \& Solberg JJ, 1987, Operational Research - Principles and practice, Wiley, New York (NY).

[3] RAO CR, 1973, Linear statistical inference and its applications, $2^{\text {nd }}$ edition, John Wiley and Sons, New York (NY).

[4] Slutsky E, 1928, Sur Les Fonctions Eventuelles Continues, Integrables et Drivables Dans le sens Stochastique, Comptes Rendus Mathematique Académie Sciences, 187, pp. $878-880$

[5] UN Fertilizer Manual, ST/CID/15, 1967, International Fertilizer Development Centre, New York (NY). 